\section{Aspergillus fumigatus as an agent of cutaneous aspergillosis in immunocompetent patient: $A$ rare case}

\author{
Trisniartami Setyaningrum, \\ Karina Dyahtantri Pratiwi \\ Department of Dermatovenereology, \\ Faculty of Medicine Airlangga \\ University, Surabaya, Indonesia
}

\begin{abstract}
Cutaneous aspergillosis occurs relatively less frequent and therefore remains poorly characterized. Previous reports have described cutaneous aspergillosis as primary or secondary infection. Primary cutaneous aspergillosis usually involves sites of skin injury, at or near intravenous access catheter sites, at sites of traumatic inoculation, and at sites associated with occlusive dressings, burns, or surgery. Primary cutaneous aspergillosis almost always seen in immunocompromised patients and the skin involvement occurs due to hematogenous dissemination from a primary focus or contiguous spread from underlying infected tissues. This is a rare case of an-56-years-oldimmunocompetence man with chief complaint of wound and swelling at left leg since 1 year ago that worsen in last 2 months. He got a history of scalded by hot water at his left leg 1 year ago and became swollen with multiple wound on its surface. Examination on regio pedis sinistra there is tumor with verrucous surface $10 \mathrm{~cm}$ in diameter, hard with multiple uneven edge ulcer $5 \mathrm{~cm}$ in diameter and hyperpigmentation macule unsharply marginated arround. Potassium hydroxide examination showed conidiophores dichotomously branching and septated hyphae that suitable with Aspergillosis sp. From cultures there is velvety-dark-green growth. The microscope findings from the culture specimen showed conidophore, metula, vesicle, phialde, and chains of pigmented conidia that suitable with Aspergillus fumigatus. Blood culture examination showed no growth of fungi. HIV rapid test negative results obtained. Patients treated with Itraconazole 2 x 200 mg for 12 weeks and obtained satisfactory result.
\end{abstract}

\section{Introduction}

Cutaneous aspergillosis may occur as either primary or secondary infection. Primary cutaneous aspergillosis usually involves sites of skin injury, at or near intravenous access catheter sites, at sites of traumatic inoculation, and at sites associated with occlusive dressings, burns, or surgery. 1,2

Numerous reports have described primary or secondary cutaneous aspergillosis in an array of non-HIV-infected immunocompromised patients, including burn victims, neonates, individuals with cancer, and bone marrow and solid-organ transplant recipients. ${ }^{2}$ Primary cutaneous infection by Aspergillus fumigatus in an immunocompetent patient is unheard of Chaturvedi et al. ${ }^{2}$

\section{Case Report}

A-56-year-old man working as a teacher for 10 years came with chief complain wound and swelling at left leg since 1 year ago that worsen in last 2 months. He felt pain and itchy sensation at the wound, and also accompanied by subfebris fever. He got a history of scalded by hot water at his left leg 1 year ago and became swollen with multiple wound on its surface. In the long run, the surface of his left leg also became verrucous.

He rubbed his left leg with oral herbal medication, minyak tawon, and sulphur. $\mathrm{He}$ already came to general practitioner and got Coamoxiclav 3x625 mg, Bactoderm Cream, $\mathrm{NaCl} 0,9 \%$ and framicetin gauze dressing (FGD) to treat the wounds but the lesion had still persists.

Patients came to Dermatovenereology Outpatient Clinic of RSUD Dr. Soetomo Surabaya. Examination on regio pedis sinistra there is tumor with verrucous surface 10 $\mathrm{cm}$ in diameter, hard with multiple uneven edge ulcer $5 \mathrm{~cm}$ in diameter and hyperpigmentation macule unsharply marginated arround. Potassium hydroxide examination showed conidiophores dichotomously branching and septated hyphae that suitable with Aspergillosis sp. Cultures examination showed the velvety-dark-green colonies growth after 14 days. The microscope findings from the culture specimen showed conidophore, metula, vesicle, phialde, and chains of pigmented conidia that suitable with Aspergillus fumigatus. Blood culture examination showed no growth of fungi. HIV rapid test negative results obtained. Patients received Itraconazole 2 x $200 \mathrm{mg}$ for 12 weeks and obtained satisfactory result (Figures 1 and 2).

\section{Discussion}

Aspergillus species is a saprophytic mold, which is found in decaying organic matter. Infection occurs by inhalation of
Correspondence: Trisniartami Setyaningrum, Department of Dermatovenereology, Faculty of Medicine Airlangga University, Surabaya, Indonesia, Mayjen. Prof. Dr. Moestopo 6-8 Surabaya 60286.

Tel.: +62811300192

E-mail: trisniartami_s@yahoo.com

Key words: cutaneous aspergillosis, Aspergillus fumigatus, Itraconazol.

Conflict of interest: the authors declare no potential conflict of interest.

Received for publication: 1 February 2019. Accepted for publication: 25February 2019.

This work is licensed under a Creative Commons Attribution-NonCommercial 4.0 International License (CC BY-NC 4.0).

(C) Copyright T. Setyaningrum and

K. Dyahtantri Pratiwi, 2019

Licensee PAGEPress, Italy

Dermatology Reports 2019; 11(s1):8089

doi:10.4081/dr.2019.8089

spores or by direct entry of organisms into body tissue through wound ${ }^{2}$.

Moisture in buildings becomes a critical factor for fungal proliferation and dissemination. Indoor dampness increases the risk of fungal growth. A complex interaction between occupant behavours and the built environment are thought to affect indoor fungal concentrations and species diversity. ${ }^{2-5}$ Primary cutaneous aspergillosis usually involves sites of skin injury at or near intravenous access catheter sites, at sites of traumatic inoculation, and at sites associated with occlusive dressings, burns, or surgery. Secondary cutaneous lesions result either from contiguous extension to the skin from infected underlying structures or from widespread blood-borne seeding of the skin. ${ }^{1}$

The skin lesions occurs as a result of direct inoculation of Aspergillus spores at the site of injury. Lesions are generally seen on extremities where they appear as erythematous papules and pustules which ulcerate to form eschar. ${ }^{1,3}$ In this patient there was a history of wound and working at a damp environment may be the source of fungal spores.

Among patients with HIV-related cutaneous aspergillosis, most common causing agent was $A$. fumigatus infection. The reason for this high proportion of primary $A$. fumigatus isolates is not known. In contrast, among cases of cutaneous aspergillosis that did not involve HIV-infected or burn patients, the following organisms accounted for the indicated proportion of cases: Aspergillus flavus, 44\%; A. fumiga- 
tus, 26\%; Aspergillus spp. (the species of Aspergillus was not determined), 10\%; Aspergillus terreus, 6\%, Aspergillus niger, 6\%, A. glaucus, 4\% Aspergillus chevalieri, 3\%; andAspergillus ustus, 1\%. Determination of the species causing aspergillosis did not guide therapy in any of the reports reviewed ${ }^{1}$. Meanwhile in this case A. fumigatus is the causing agent in an immunocompetent individual.

A presumptive diagnosis of primary cutaneous aspergillosis can be made immediately by examining a potassium hydroxide preparation of a biopsy specimen. The diagnosis of most primary Aspergillus infections requires biopsy of a skin lesion taken for both culture and histopathology. A skin biopsy specimen for a suspected fungal lesion should be taken from the center of the lesion and should reach the subcutaneous fat because Aspergillus tends to invade blood vessels of the dermis and subcutis, resulting in an ischemic cone above it. ${ }^{1,3}$

Fungal isolates from culture media are identified on the basis of colony morphology, color, and sporulation. The specimen should be minced and plated on medium specific for the recovery of yeast (e.g., bromcresol green), mold (e.g., potato dextrose agar), and dermatophytes (e.g., Mycobiotic) and should be held for 6 weeks. The variations of colony morphology in Aspergillus are many, depending upon the species. Most species begin as white colonies, but rapidly develop colors of green, yellow, orange, black or brown. Colonies are fluffy to velvet like and are mature in 3-5 days. Some form concentric circles of color while others are uniform in color. $^{2,6}$

The appearance of hyphae with acuteangle branching alone, however, is not specific enough to distinguish Aspergillus hyphae from other those of other medically important filamentous molds such as Pseudallescheria boydii and Fusarium spp. In addition, with certain angles of specimen sectioning, acuteangle Aspergillus branches may appear as right-angle branches, thus resembling the right-angle branching of pauciseptated hyphae of zygomycete-like species. ${ }^{1,3}$

The long conidiophores arise from hyphae via a foot cell, ending in a swollen vesicle. Some environmental species form hyphae that are spiral or zig-zag in appearance. All form a vesicle, that may be round, semi-circular or club shaped. The vesicle becomes covered with phialides, occurring singly (seriate) or in pairs (biseriate) connected end to end. From the phialides emerge the conidia that form chains. The conidia are round with a smooth or rough surface. Morphologically, the arrangement of the phialides and their orientation on the vesicle can be used to help identify the various species. ${ }^{6}$

Itrakonazole has been used for the treatment of cutaneous aspergillosis. Its mode of action is through the inhibition of the cytochrome P-450-dependent demethylation stage in the formation of ergosterol on the fungal cell membrane. Itraconazole is well absorbed orally, and because of its highly lipophilic character, it is accumulat- ed in the tissue at a higher level than in the plasma. The bioavailability of the drug is increased if it is taken with a fatty meal, but can be decreased in patients taking drugs that impair gastric acidity, such as histamine- 2 blockers and antacids. ${ }^{6-9}$ There is no difference treatment of cutaneous aspergillosisn in an immunocompetence and immunocompromised patients. In this case, there was a good clinical response of Itrakonazole treatment.

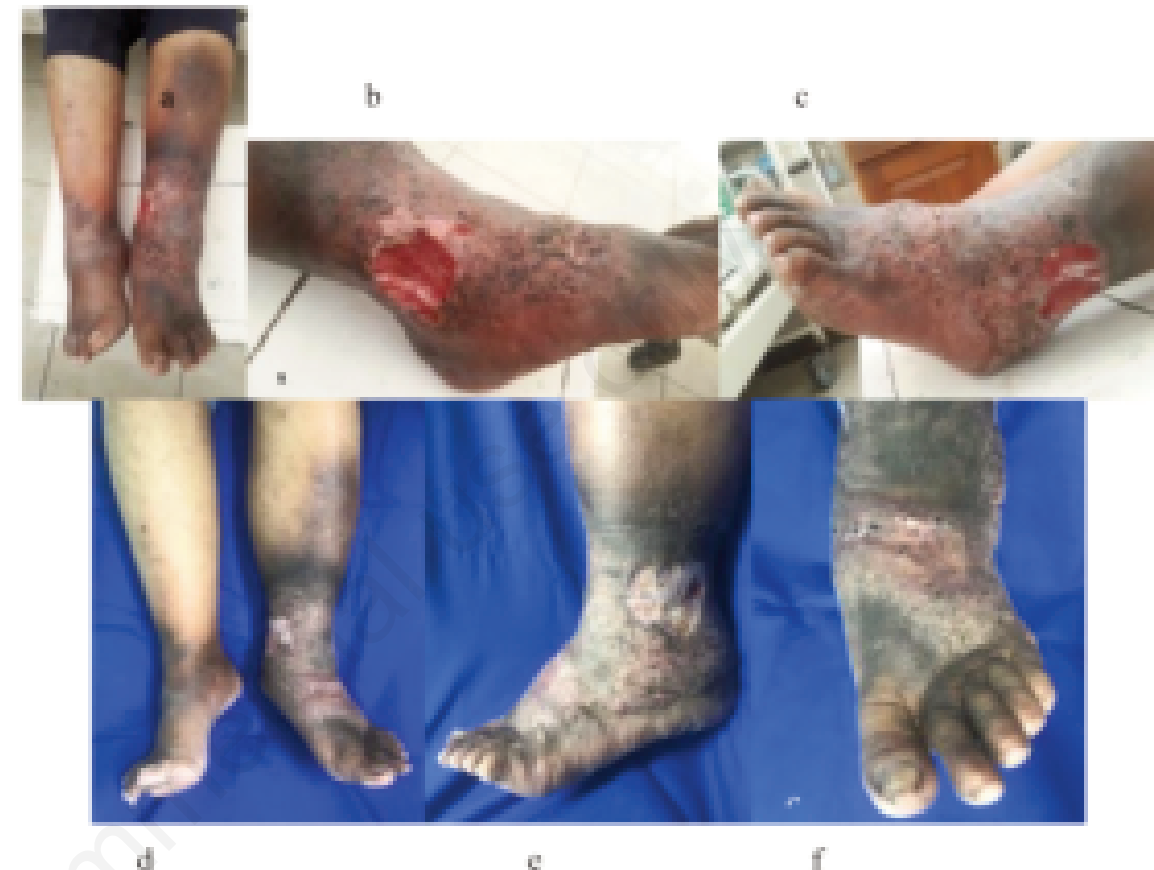

Figure 1. a, b, c Tumor with verrucous surface $10 \mathrm{~cm}$ in diameter, hard with multiple uneven edge ulcer $5 \mathrm{~cm}$ in diameter and hyperpigmentation macule unsharply marginated around at regio pedis sinistra; d, e, f After treatment with Itraconazole 2 × $200 \mathrm{mg}$ for 12 weeks. Verroucous surface has been decreased and wound healing showed a good pregress.
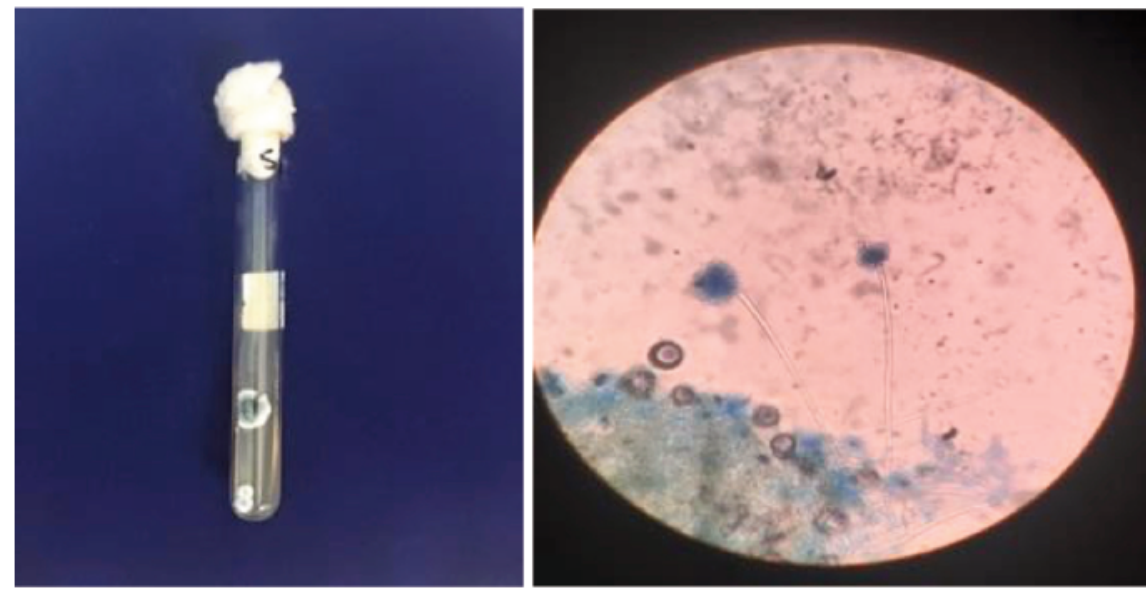

Figure 2. Velvety-dark-green colonies growth on cultures examination after 14 days. Microscopic findings from the culture specimen showed conidophore, metula, vesicle, phialde, and chains of pigmented conidia that suitable with Aspergillus fumigatus. 


\section{Conclusions}

Primary cutaneous aspergillosis is a condition associated with extensive morbidity and mortality. Dissemination of immunocompromised status were significantly associated with increased mortality. Early diagnosis of primary cutaneous aspergillosis is key to improved outcomes. A case of primary cutaneous aspergillosis in an immunocompetent patient is unheard of, but happened in this case by the basic principal of host-agent-environment condition.

\section{References}

1. Burik. Cutaneous Aspergillosis. J. Clin. Microbiol 1998; 36(11): 3115-22

2. Chaturvedi, R., Kolhe, A., Pardeshi K, Naik L., Wanjare, S. Primary cutaneous aspergillosis, mimicking malignancy, a rare presentation in an immunocompetent patient. Diagnostic Cytopathology 2017; 1-4.

3. Darr-Foit, S. Primary cutaneous aspergillosis-an uncommon opportunistic infection. Journal of the German Society of Dermatology 2017; 839-41

4. Moore, R., Lopes, J. Paper templates. In TEMPLATE'06, 1st International Conference on Template Production 1999; SCITEPRESS.
5. Richardson M. Mycoses: Indoor fungal exposure and disease: an overview. Blackwell Verlag GmbH 2015; 58 (Suppl. 4), 14-19

6. Sciortino. Atlas of Clinically Imporant Fungi: Hyaline molds Aspergillus spp. Wiley: New Jersey. 2017;p167-170

7. Sigurgeirsson, B., Hay, R.J., Antibiotics and antifungal therapy in dermatology: The antifungal drugs used in skin disease. Springer International Publishing: Switzerland.p147-148

8. Smith, J. The book, The publishing company. London 1998; 2nd edition

9. Tatara, A.M., Mikos, A.G., Kontoyiannis, D.P. Factors affecting patient outcome in primary cutaneous aspergillosis. Medicine (United States) 95. .2016 